\title{
Die (Friedens-)Pfeife
}

\author{
Erhard Taverna
}

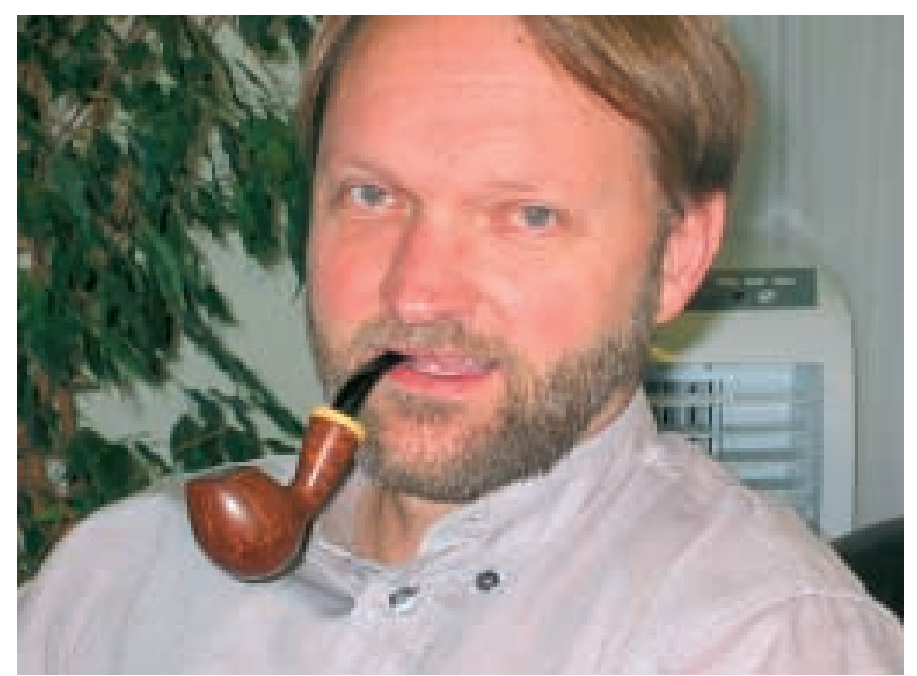

Für Friedensabschlüsse, Geschäftsverträge und Freundschaftserklärungen war die Calumet-Zeremonie den Indianern unentbehrlich. Dazu gehörten der Pfeifenkopf aus rotem Tonschiefer vom Steinbruch aus Minnesota und das Rohr aus Weide oder Esche, mit Federn oder Perlen verziert. Heute ist alles viel besser, nicht der Frieden, aber das Material, die reiche Auswahl und ein Tabak, der nicht mehr mit Bärentrauben und Rindenstücken vermischt wird.

\section{Der Macher}

Einer, der das Handwerk auch beherrscht, ist der 1958 geborene Daniel Jud, Psychiater FMH mit Praxis in Amriswil TG, verheiratet, zwei Kinder. Mit einem Bastlergen vom Vater ist er früh auf den Geschmack gekommen, hat die weggeworfene Pfeife des Nachbarn instandgestellt und mit trockenen Heusamen gestopft. Zu besseren Modellen und Mischungen kam er im Verlauf des Gymnasiums und Medizinstudiums. Sein manuelles Geschick als Assistenzarzt in der Psychiatrischen Klinik muss aufgefallen sein, denn ein Maltherapeut riet ihm zur Eigenkonstruktion. So kamen aus Hamburg die ersten vorgebohrten Rohlinge mit fertigem Mundstück. Daniel Jud war mit dem Herausfeilen der gewünschten Formen derart erfolgreich, dass er sich an unbearbeitete Blöcke wagte und Mundstücke aus Ebonitstangenmaterial fertigte. Der Profi braucht dazu eine Drehbank und ein Dreibackenfutter. Geraspelt wird von Hand oder mit der Tellerschleifscheibe mit Absaugvorrichtung. Anfangs dauerte das vier bis fünf Stunden pro Pfeife, heute braucht er für das einfachste Modell etwa zwei bis drei, für eine anspruchsvolle Arbeit kann es auch bis fünfzehn Stunden dauern.

Das Bruyèreholz stammt aus dem Mittelmeerraum und ist die Wurzelknolle der Erica arborea. Er bezieht sein Holz aus Spanien, andere schwören auf die korsische oder algerische Herkunft. Ton, Meerschaum oder Glas ist nicht sein Ding. Der Meister konzentriert sich auf den gewachsenen Stoff mit einem Mundstück aus Parakautschuk, das er dem weniger bissfreundlichen Akrylharz vorzieht. Entscheidend für die Qualität ist ein runder Pfeifenboden mit einem Rauchkanal, der genau an der richtigen Stelle ansetzt. Wird der Boden zu spitz, verbrennt der Tabak schlecht, wird er zu flach, gibt es einen schlechten Zug. Dazu braucht es eine ausgetüftelte Bohrung des Holms und der Brennkammer und eine künstlerische Hand für die Holmringe und Zapfen aus tropischen Edelhölzern, Buchsbaum oder Metall. Die Wuchsrichtung bestimmt die Maserung des Pfeifenkopfes, aus weichen und harten Stellen werden beim Sandstrahlen ringartige Strukturen. Dann wird das Kolbeninnere mit Wasserglas und Holzkohlenpulver imprägniert, Name und Datum angebracht und fertig ist die Friedenspfeife.

\section{Die Geniesser}

Seit sich Daniel Jud vor zwei Jahren über ebay.de als Pfeifenkonstrukteur geoutet hat, wächst seine weltweite Fangemeinde von Sammlern, Händlern, Konsumenten und anderen Künstlern. Seine 30-40 Exemplare jährlich vertreibt er über Direktverkauf oder seinen Händler in Brissago und Schweden zum Ladenpreis von 300 bis 350 Franken das Stück. Wenig im Vergleich zu einer Luxuspfeife von z.B. Bo Nordh aus Schweden, die einige tausend Franken kosten kann. Freehands und Anfertigungen nach den Vorstellungen von Pfeifenrauchern, wie z.B. eine Standup-Poker-Pfeife mit rundum vorhandener Bird-Eyes-Maserung, liegen durchaus drin, doch er selber bevorzugt die Klassiker der 20-30 existierenden Grundmodelle. 
Sein eigenes Rezept für einen friedlichen Tag verrät er gerne: mittags eine englische Mischung mit Latakia-Zusatz, Brenndauer etwa eine dreiviertel Stunde. Abends einen Flaketabak, etwas rauchiger, mit gepresstem, in Scheiben geschnittenem virginiaversetztem Tabak.

Anfängern rät er zu einer neutralen Sorte, nicht zu fein geschnitten, da sonst die Pfeife zu heiss wird. Von Anfang an vollstopfen und die heisse Zunge als Initiationsphase hinter sich bringen.
Pfeifenraucher gebe es heute mehr als noch vor einigen Jahren, findet Jud. Auch als Psychiater kann er keinen idealtypischen Konsumenten ausmachen. Zwar haben noch wenige Frauen zum traditionellen Phallusobjekt gefunden, doch Pfeifenmacherinnen kenne er bereits zwei. Pfeifenraucher setzten auf Qualität und Zeit. Sie sind Genussmenschen wie die Weinliebhaber. Zeit, Qualität und Genuss im neuen Jahr wünsche ich auch allen Leserinnen und Lesern der Schweizerischen Ärztezeitung.

\title{
Diagnoseprozesse und Wissenssysteme
}

\author{
Tagung am Collegium Helveticum in Zürich vom 21./22. Januar 2005
}

\author{
G. Schönbächler
}

Korrespondenz:

Dr. sc. nat. Georg Schönbächler Collegium Helveticum

STW/C12.2

Schmelzbergstrasse 25

CH-8092 Zürich

Tel. 0446323385

Fax 0446321204

E-Mail:

schoenbaechler@collegium.ethz.ch
Die Wolcke und das Sediment im Urin in hitzigen Fiebern ist ein vorbedeutendes Zeichen, dass eine Crisis erfolgen wird, folglich ein signum prognosticum criseos.

Ernst Anton Nicolai. Samuel Schaarschmidts Semiotic oder Lehre von den Kennzeichen des innerlichen Zustandes des menschlichen Körpers mit Zusätzen vermehret (1756)

Die Semiotik, die Lehre von den Zeichen, bildete bis in die Neuzeit eine bedeutende medizinische Grundlagenwissenschaft. Der französische Ausdruck «semiologie» (Symptomatologie) deutet noch heute darauf hin. Die Erfolge der naturwissenschaftlichen Methoden und die damit zusammenhängenden Fortschritte in der Medizin haben in den vergangenen zwei Jahrhunderten die Semiotik als eigenständigen medizinischen Wissenschaftszweig verdrängt und die enge Verwandtschaft zu dieser Disziplin immer mehr in den Hintergrund treten lassen.

Zwar werden auch in der naturwissenschaftlichen Medizin semiotische Begriffe wie «Rezeptor», «Transmitter», «Signalübertragung» oder «genetischer Kode» verwendet, doch werden Er- kenntnisse aus der Semiotik für medizinische Zwecke mit Ausnahme des biosemiotischen Ansatzes des kürzlich verstorbenen Thure von Uexküll kaum genutzt.

Aber nicht nur bei der Beschreibung metabolischer Abläufe im Körper können semiotische Kategorien hilfreich sein, auch bei der Tätigkeit des Diagnostizierens handelt es sich primär um einen Zeichenprozess: Ein Symptom als Verhaltensauffälligkeit des menschlichen Körpers verweist auf eine zugrundeliegende, unter Umständen behandlungsbedürftige Krankheit und ist insofern ein Zeichen.

Diesen Zeichenprozessen ist die Tagung «Diagnoseprozesse und Wissenssysteme» gewidmet. Die Veranstaltung versucht, dem semiotischen Ansatz Rechung zu tragen, und nähert sich dem Thema immer vor dem Hintergrund der Medizin auf verschiedenen Ebenen an: von der Agrikultur über die Medizininformatik, Psychiatrie, Alchemie, Kunstgeschichte bis hin zur Literatur. Die Tagung zielt insbesondere darauf ab, den wissenschaftshistorisch zwar erklärbaren, aber einschränkenden Graben zwischen Semiotik und Medizin zu verkleinern und damit einen Beitrag zu leisten zur Verständigung unterschiedlicher Wissenschaftsdisziplinen. 


\section{Tagungsprogramm}

Freitag, 21. Januar 2005

Chair: Dr. Barbara Orland

15.00 Eröffnung der Tagung und Begrüssung Prof. Dr. Gerd Folkers, Leiter Collegium Helveticum

15.15 Zur Einführung: Die Diagnose als Zeichenprozess Dr. Georg Schönbächler, Collegium Helveticum

15.30 Intentionalität und Kausalität bei Zeichenprozessen Prof. Dr. Roland Posner, Arbeitsstelle für Semiotik, FU Berlin

16.15 Pause

16.45 Diagnosekodierung als Interpretation sprachlicher Zeichen Dr. med. Hans Rudolf Straub, Semfinder AG, Kreuzlingen

17.30 Bodenbeurteilung zwischen Praxis und Wissenschaft Dr. Patricia Fry, Wissensmanagement im Umweltbereich, Zürich

18.15 Pause

18.30 Diagnose subjektiver Strukturen Dr. Klaus Leferink, Berlin

19.15 Abendessen im Café Semper

20.15 Nicht das Gehirn, sondern der Mensch fühlt und denkt Prof. Dr. Daniel Hell, Psychiatrische Universitätsklinik Burghölzli, Zürich

21.00 Ausklang im Café Semper

Samstag, 22. Januar 2005

Chair: Prof. Dr. Gerd Folkers

$9.00 \quad$ Typen begrifflicher Ordnungssysteme in der Medizin Dr. Josef Ingenerf, Universität Lübeck

9.45 Wie Zeichen sprechen Prof. Dr. Johannes Fehr, Collegium Helveticum

10.30 Pause

$11.00 \quad$ Die Krankheit im Spiegel Dr. Georg Schönbächler, Collegium Helveticum

11.45 Naturbeobachtung und Mythologie als Grundlagen ganzheitlicher Diagnostik und Therapie bei Theophrast von Hohenheim (Paracelsus) Dr. Pirmin Meier, Beromünster

Schlussdiskussion: Rückblick und Ausblick

$13.00 \quad$ Abschiedslunch im Café Semper 